Classification

Physics Abstracts

$03.20-64.00-61.70$

\title{
Metastability and possible failure of iteration methods
}

\author{
A. Engel \\ Sektion Physik der Humboldt-Universität, 1040 Berlin, G.D.R. \\ (Reçu le 27 novembre 1984, révisé le 19 février 1985, accepté le 7 mars 1985)
}

\begin{abstract}
Résumé. - On applique à un modèle simple de potentiel aléatoire la méthode d'itération proposée par Efetov et Larkin. On démontre que le paramètre de développement de cette méthode est du même ordre de grandeur que le nombre des états métastäbles. Si ce nombre est grand, comme c'est le cas pour un système dans un potentiel aléatoire, la méthode correspond à une moyenne blanche sur tous les extrema de l'Hamiltonien.
\end{abstract}

\begin{abstract}
The applicability of an iteration method as proposed by Efetov and Larkin to a simple model of a random field system is investigated. It is shown that the expansion parameter of the method is of order of the number of metastable states. If this number is large, as is typical for random field systems, the method produces white averages over all extrema of the Hamiltonian.
\end{abstract}

\section{The model.}

The description of the ground state $(T=0)$ properties of a random field system involves the determination of the configuration minimizing the Hamiltonian for a given realization of the randomness. Sometimes [1,2] this is attempted by solving the corresponding Euler-Lagrange equation (ELE) by an iteration procedure in the random field strength as proposed by Efetov and Larking in the context of charge density waves [3]. The great advantage of this method is that it yields exact results for averaged quantities by its first order term since higher order contributions cancel exactly after averaging over the random field [3].

On the other hand Villain and Séméria [4] have recently drawn attention to possible dangers of such methods. For the problem of interfaces in random fields it is, for example, well known that several averaged ground state quantities depend nonanalytically on the disorder strength [4-8] which makes the appropriateness of iteration methods questionable. Furthermore there are in general many solutions of the ELE and there is no guarantee that the iteration method indeed picks the minimum of the Hamiltonian.

In order to investigate these problems we discuss in this note the application of the iteration method to a simple 1D random field system given by the Hamiltonian

$$
H(f)=H_{\mathrm{e}}(f)+H_{\mathrm{r}}(f)=\frac{a}{2} f^{2}-h \int_{0}^{f} \varepsilon(z) \mathrm{d} z
$$


Here $a$ is a constant and $\varepsilon(z)$ is a random field with Gaussian statistics given by

$$
\overline{\varepsilon(z)}=0, \quad \overline{\varepsilon(z) \varepsilon\left(z^{\prime}\right)}=K\left(\left|z-z^{\prime}\right|\right)=\frac{1}{\Delta} \mathrm{e}^{-\frac{\left(z-z^{\prime}\right)^{2}}{2 \Delta^{2}}}
$$

where we have introduced the correlation length $\Delta$. At $T=0$ the elastic term $H_{\mathrm{e}}$ tries to make $f=0$ whereas the random field term $H_{\mathrm{r}}$ describes possible energy gain from the random field for $f \neq 0$. The model has been introduced by Villain et al. [9]. It is not only interesting in itself but also emerges in the renormalization group treatment of the random field Ising model [5, 6] where $f$ gives the amplitude of a wavepacket contribution to the interface configuration on a length scale $L$. In this case $a$ is of order $L^{\frac{d-5}{2}}$.

The ELE corresponding to equation (1) is

$$
a f=h \varepsilon(f)=h \int \frac{\mathrm{d} q}{2 \pi} \varepsilon_{q} \exp \{\text { iqf }\}
$$

with $\varepsilon_{q}$ being the Fourier transform of $\varepsilon(z)$, therefore obeying the statistics

$$
\overline{\varepsilon_{q}}=0, \quad \overline{\varepsilon_{q} \varepsilon_{q^{\prime}}}=2 \pi \delta\left(q+q^{\prime}\right) K_{q}=(2 \pi)^{3 / 2} \delta\left(q+q^{\prime}\right) \mathrm{e}^{-\frac{\Delta^{2} q^{2}}{2}}
$$

We will solve equation (2) by iteration and discuss whether or not the solution $f_{\text {it }}$ obtained coincides with the ground state $f_{\mathrm{G}}$ minimizing (1).

\section{The iteration solution.}

In order to solve equation (2) by iteration we follow Efetov and Larkin [3] and assume that $f$ can be written as a power series in $h$ :

$$
f=\sum_{i=1}^{\infty} h^{i} f_{i}
$$

Inserting this ansatz in (2) and expanding the exponent we find :

$$
a \sum_{i=1}^{\infty} h^{i} f_{i}=h \int \frac{\mathrm{d} q}{2 \pi} \varepsilon_{q} \exp \left\{i q \sum_{i=1}^{\infty} h^{i} f_{i}\right\}=\sum_{i=0}^{\infty} h^{i+1}\left[\int \frac{\mathrm{d} q}{2 \pi} \varepsilon_{q} \sum_{\substack{\left\{k_{j}\right\}, j \\ \sum_{j} j-k_{j}=i}} \frac{(i q)_{j} \boldsymbol{k}_{j}}{\prod_{j}\left(k_{j} !\right)} \prod_{j} f_{j}^{\boldsymbol{k}_{j}}\right] .
$$

It is convenient to write the coefficients on the right hand side as tree diagrams of the type used in [3]. Matching powers of $h$ on both sides of (4) the $f_{i}$ can be determined one after another.

On the other hand a simple scaling argument applied to (2) yields

$$
\overline{f^{2}}=\Delta^{2} F\left(\frac{h}{a \Delta^{3 / 2}}\right)
$$

where $F$ is an unknown function. Indeed inspection of the first $f_{i}$ reveals that equation (4) gives, for typical realizations of the random field,

$$
\frac{f_{i}}{\Delta} \sim\left(a \Delta^{3 / 2}\right)^{-i}
$$


in accordance with (5) and the fact that $\frac{h}{a \Delta^{3 / 2}}$ is the only dimensionless parameter of the model proportional to $h$. Hence instead of (3) it is more appropriate to use the ansatz

$$
f=\Delta \sum_{i=1}^{\infty} \tilde{f_{i}}\left(\frac{h}{a \Delta^{3 / 2}}\right)^{i}
$$

where now the $\tilde{f}_{i}$ depend only on $i$. Note that $\frac{h}{a \Delta^{3 / 2}}$ can be large for $\Delta \rightarrow 0$ or $a \rightarrow 0$ (as a result of $L \rightarrow \infty$ and $d<5$ ) even if $h$ is small.

It is useful now to look at a graphical solution of (2) (Fig. 1). Typical values of $\varepsilon(z)$ lie between $\pm \Delta^{-1 / 2}$, therefore most of the solutions of equation (2) lie within the interval $\left(-f_{\max }, f_{\max }\right)$ with

$$
f_{\max } \sim \frac{h}{a \Delta^{1 / 2}}
$$

The difference between any two successive solutions is of order $\Delta$, therefore

$$
N \sim \frac{h}{a \Delta^{3 / 2}}
$$

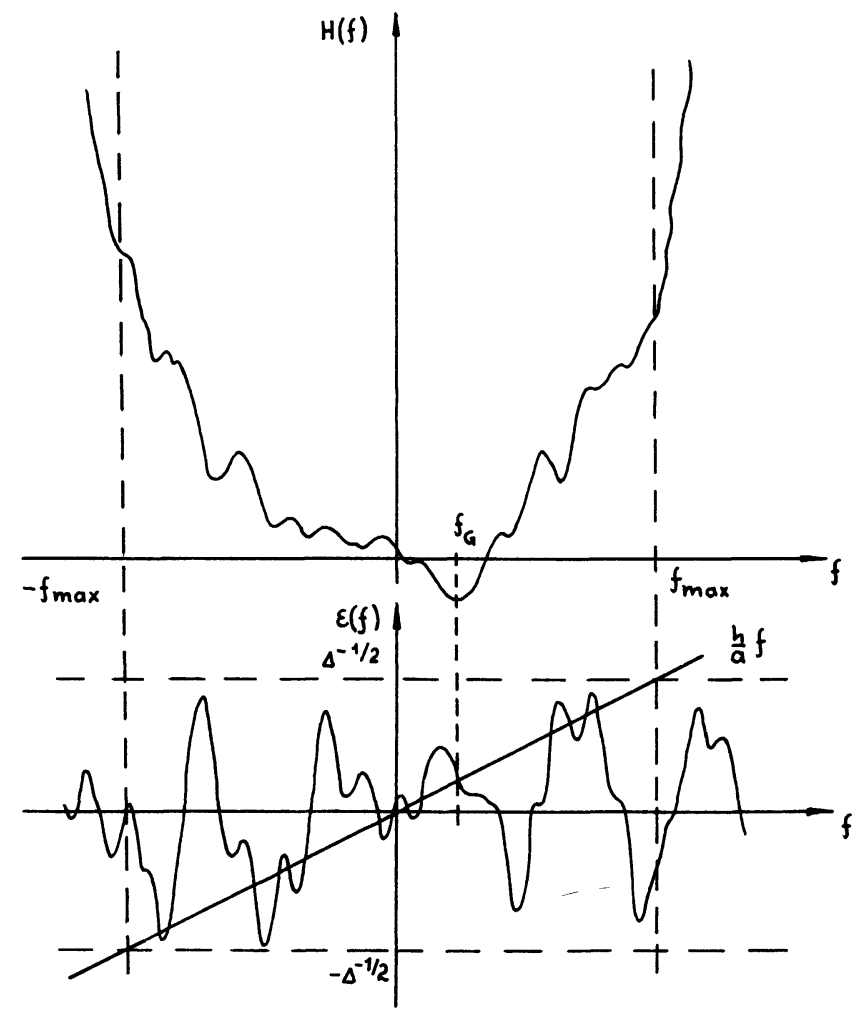

Fig. 1. - Qualitative plot of the function $H(f)$ given by equation (1) and graphical solution of the corresponding Euler-Lagrange equation. 
gives the number of possible solutions of equation (2). It is just this number which serves as expansion parameter in (6). We discuss the result of the iteration method now for the two cases $N \ll 1$ and $N \gg 1$ separately.

\section{3. $N \ll 1$.}

No problems arise in this case. The ELE (2) has, for typical realization of the randomness, only one solution which is necessarily $f_{\mathrm{G}}$ and on the other hand series (6) is expected to converge. As in the case of Efetov and Larkin [3] we find

$$
\overline{f^{n}}=h^{n} \overline{f_{1}^{n}}
$$

i.e. higher order contributions cancel after averaging. Computing $\overline{f^{2}}$ we get, for example, for the term proportional to $h^{4}$ :

$$
\overline{f_{2}^{2}}+2 \overline{f_{1} f_{3}}
$$

From (4) it follows that

$$
\begin{aligned}
& f_{1}=\frac{1}{a} \int \frac{\mathrm{d} q}{2 \pi} \varepsilon_{q} \\
& f_{2}=\frac{1}{a^{2}} \int \frac{\mathrm{d} q}{2 \pi} \int \frac{\mathrm{d} q^{\prime}}{2 \pi} i q \varepsilon_{q} \varepsilon_{q^{\prime}} \\
& f_{3}=\frac{1}{a^{3}} \int \frac{\mathrm{d} q}{2 \pi} \int \frac{\mathrm{d} q^{\prime}}{2 \pi} \int \frac{\mathrm{d} q^{\prime \prime}}{2 \pi}\left[i q \varepsilon_{q} i q^{\prime} \varepsilon_{q^{\prime}} \varepsilon_{q^{\prime \prime}}+\frac{(i q)^{2}}{2} \varepsilon_{q} \varepsilon_{q^{\prime}} \varepsilon_{q^{\prime \prime}}\right] .
\end{aligned}
$$

Therefore we find

$$
\begin{aligned}
\overline{f_{2}^{2}} & =\frac{1}{a^{4}} \int \frac{\mathrm{d} q}{2 \pi} \int \frac{\mathrm{d} q^{\prime}}{2 \pi} q^{2} K_{q} K_{q^{\prime}} \\
\overline{f_{1} f_{3}} & =\frac{1}{a^{4}} \int \frac{\mathrm{d} q}{2 \pi} \int \frac{\mathrm{d} q^{\prime}}{2 \pi} q^{2} K_{q} K_{q^{\prime}}-\frac{3}{2 a^{4}} \int \frac{\mathrm{d} q}{2 \pi} \int \frac{\mathrm{d} q^{\prime}}{2 \pi} q^{2} K_{q} K_{q^{\prime}}
\end{aligned}
$$

and hence

$$
\overline{f_{2}^{2}}+2 \overline{f_{1} f_{3}}=0 \text {. }
$$

Using the diagrammatic representation of (4) and arguments similar to those of reference [3] one can show that these cancellations hold for all higher order terms.

Therefore we find in particular :

$$
\overline{f_{\mathrm{G}}^{2}}=h^{2} \overline{f_{1}^{2}}=\frac{h^{2}}{a^{2} \Delta} \sim N^{2} \cdot \Delta^{2}
$$

where we have used (4) and (7). For $N \ll 1$ we have therefore $\overline{f_{G}^{2}} \ll \Delta^{2}$. The results obtained are in agreement with those produced by energy arguments similar to Imry and $\mathrm{Ma}[10,11]$. Indeed for $\left|f_{\mathrm{G}}\right| \ll \Delta$ we find

$$
H(f) \sim \frac{a}{2} f^{2}-h \frac{f}{\Delta^{1 / 2}}
$$

and the minimum of $H$ is realized for

$$
f_{\mathrm{G}} \sim \frac{h}{a \Delta^{1 / 2}}
$$


4. $N \gg 1$.

Now the ELE (2) has many solutions as shown in figure 1 . The iteration solution $f_{\text {it }}$ is once more given by (6) and operating formally as in the case $N \ll 1$ we find again

$$
\overline{f_{\mathrm{it}}^{2}}=h^{2} \overline{f_{1}^{2}}=\frac{h^{2}}{a^{2} \Delta} \text {. }
$$

However one now expects that the series (6) will diverge for typical disorder realizations. It makes no sense to average a divergent series term by term, so the cancellation of higher order terms cannot be used now without further justification. For this purpose it would be useful to show that (6) can be summed by some resummation technique even for $N \gg 1$.

However we find it more transparent to use the following path-integral expression for the probability distribution $P(f)$ of $f[12]$ to clarify the meaning of $(8)$ :

$$
P(f)=\text { const. } \times \int \mathscr{D} \varepsilon \delta\left(f-\frac{h}{a} \varepsilon(f)\right)\left|1-\frac{h}{a} \varepsilon^{\prime}(f)\right| P[\varepsilon] .
$$

The constant in (9) ensures the normalization of $P(f)$ and can be determined at the end. Integrating (9) over $f$ one realizes that it will depend on the average number of zeros of the argument of the $\delta$-function.

$P[\varepsilon]$ is given by

$$
P[\varepsilon]=\frac{1}{\mathcal{N}} \exp \left\{-\frac{1}{2} \int \mathrm{d} z \int \mathrm{d} z^{\prime} \varepsilon(z) K^{-1}\left(z, z^{\prime}\right) \varepsilon\left(z^{\prime}\right)\right\}
$$

where $\mathcal{N}$ is the proper normalization constant.

Using now

and

$$
\delta(x)=\int \frac{\mathrm{d} q}{2 \pi} \mathrm{e}^{i q x}
$$

we find

$$
|x|=\int \frac{\mathrm{d} k}{\pi} \frac{\sin ^{2} k x}{k^{2}}
$$

$$
\begin{aligned}
P(f)=\text { const. } \times \int \frac{\mathrm{d} q}{2 \pi} \int \frac{\mathrm{d} k}{2 \pi} \mathrm{e}^{i q f} \frac{1}{2 k^{2}} \frac{1}{\mathcal{N}} \int \mathcal{D} \varepsilon \exp \left\{-i q \frac{h}{a} \varepsilon(f)-\right. \\
\left.-\frac{1}{2} \int \mathrm{d} z \int \mathrm{d} z^{\prime} \varepsilon(z) K^{-1}\left(z, z^{\prime}\right) \varepsilon\left(z^{\prime}\right)\right\}\left[2-\mathrm{e}^{2 i k\left(1-\frac{h}{a} \varepsilon^{\prime}(f)\right)}-\mathrm{e}^{-2 i k\left(1-\frac{h}{a} \varepsilon^{\prime}(f)\right)}\right] .
\end{aligned}
$$

Now the path-integral can be performed yielding

$$
\begin{aligned}
& P(f)=\text { const. } \times \int \frac{\mathrm{d} q}{2 \pi} \int \frac{\mathrm{d} k}{2 \pi} \mathrm{e}^{i q f} \frac{1}{2 k^{2}} {\left[2-\left(\mathrm{e}^{2 i k+2 q k C(f)}+\mathrm{e}^{-2 i k-2 q k C(f)}\right)\right] \times } \\
& \times \exp \left\{-\frac{h^{2}}{2 a^{2}}\left(q^{2} K(f, f)+\left.4 k^{2} \frac{\partial^{2}}{\partial z \partial z^{\prime}} K\left(z, z^{\prime}\right)\right|_{z=z^{\prime}=f}\right)\right\}
\end{aligned}
$$

with

$$
C(f)=-\frac{h^{2}}{2 a^{2}}\left(\left.\frac{\partial}{\partial z^{\prime}} K\left(f, z^{\prime}\right)\right|_{z^{\prime}=f}+\left.\frac{\partial}{\partial z} K(z, f)\right|_{z=f}\right) .
$$


For a macroscopically homogeneous random field ensemble we have $K\left(z, z^{\prime}\right)=K\left(\left|z-z^{\prime}\right|\right)$. Therefore

and we are left with

$$
\begin{gathered}
C(f)=0 \\
K(f, f)=K(0)=\frac{1}{\Delta} \\
\left.\frac{\partial^{2}}{\partial z \partial z^{\prime}} K\left(z, z^{\prime}\right)\right|_{z=z^{\prime}=f}=-\left.\frac{\partial^{2}}{\partial z^{2}} K(z)\right|_{z=0}=\frac{1}{\Delta^{3}}
\end{gathered}
$$

$$
P(f)=\text { const. } \times \exp \left\{-\frac{1}{2} \frac{a^{2} \Delta}{h^{2}} f^{2}\right\} \int \frac{\mathrm{d} k}{2 \pi} \frac{1-\cos 2 k \mathrm{e}^{-2 N^{2} k^{2}}}{k^{2}} .
$$

The $k$-integral only influences the normalization of $P(f)$, it is of order 1 for $N \ll 1$ and of order $N$ for $N \gg 1$ as expected from (9). Hence $P(f)$ is Gaussian with

$$
\bar{f}=0, \quad \overline{f^{2}}=\frac{h^{2}}{a^{2} \Delta} .
$$

This result is interesting for two reasons. First we see that it is the macroscopic homogeneity of the random field ensemble which is the symmetry responsible for the somewhat unexpected cancellation of higher order terms of the iteration method after averaging (which corresponds to the Gaussian character of $P(f)$ ). The well-known fact that a random field ensemble has in general a higher symmetry than a single realization of it shows up here in a remarkable simplification of averaged in comparison with unaveraged quantities.

Secondly we get from (9)

$$
P(f) \sim \int \mathscr{D} \varepsilon \sum_{\alpha=1}^{N(\varepsilon)} \delta\left(f-f_{\alpha}(\varepsilon)\right) P[\varepsilon]
$$

where $f_{\alpha}$ denotes the solutions of (2); i.e. the result (10) is only obtained, if we include all solutions of the ELE(2) with the same weight.

Therefore the result (8) of the iteration method can be interpreted as an average over all solution of the ELE. For $N \gg 1$ there are many such solutions, mostly metastable states and even maxima of the Hamiltonian, so that the iteration method in this case fails to yield the characteristics of the ground state $f_{\mathbf{G}}$. We get an estimate for $\overline{f_{\mathrm{G}}^{2}}$ using once more the energy arguments. Now we have $\left|f_{\mathrm{G}}\right| \gg \Delta$ and find $[10,11]$

$$
H(f) \sim \frac{a}{2} f^{2}-h f^{1 / 2}
$$

and minimization yields

$$
\overline{f_{\mathrm{G}}^{2}} \sim\left(\frac{h}{a}\right)^{4 / 3} \sim \frac{h^{2}}{a^{2} \Delta} N^{-2 / 3}
$$

As expected we have $\overline{f_{\mathrm{G}}^{2}} \ll \overline{f_{\mathrm{it}}^{2}}$. This is also evident from a comparison of the distribution (10) and the corresponding distribution for $f_{\mathrm{G}}$ which has been obtained by Villain et al. [9] and which reads in our notation

$$
P\left(f_{\mathrm{G}}\right) \sim \exp \left\{-\frac{a^{2}}{2 h^{2}}\left|f_{\mathrm{G}}\right|^{3}\right\}
$$


Note furthermore that (11) corresponds to the choice $F(x)=x^{4 / 3}$ in (5) which is the only form of $F$ making $\overline{f^{2}}$ independent of $\Delta$ (which is reasonable for the ground state $f_{\mathrm{G}}$ ) whereas (8) corresponds to $F(x)=x^{2}$ thereby retaining a dependence on $\Delta$.

\section{Discussion.}

Since it is well known that random field systems possess a large number of metastable minima of the Hamiltonian, then it is the case $N \gg 1$ which is typical for such systems. As shown above for this case the iteration method does not produce the ground state $f_{\mathrm{G}}$ but rather a white, that is unweighted average over all extrema of the Hamiltonian. Accordingly $f_{\text {it }}$ has properties very different from $f_{\mathrm{G}}$, for example $\overline{f_{\text {it }}^{2}} \rightarrow \infty$ for $\Delta \rightarrow 0$. We believe this to be true also for other models in higher dimension to which our model is closely related via renormalization group procedures. The failure of the iteration method in the case of strong disorder (corresponding to $N \gg 1$ ) has already been anticipated by Villain and Séméria [4]. Studying the low-temperature behaviour of the random field Ising model, Bray [13] has shown that the loop expansion for the free energy diverges and that truncation of the expansion after the one-loop contribution yields unphysical results. He relates these problems to the existence of metastable states with long relaxation times in close analogy with our findings. Furthermore Parisi and Sourlas [14] found that the existence of several solutions of a stochastic differential equation entails the spontaneous breakdown of hidden supersymmetry typical for these equations in the case where the solution is unique.

As has been pointed out recently [14-16] the relaxation from metastable states to the ground state can be very slow and results of the iteration procedure are possibly in good agreement with experiments performed on systems trapped in metastable states.

\section{Acknowledgments.}

I am very much indebted to Dr. T. Nattermann for suggesting the problem to me and for numerous helpful advice. It is a pleasure to thank Prof. A. S. Patashinski for a valuable remark and Prof. W. Ebeling for a critical reading of the manuscript.

\section{References}

[1] Dotsenko, V. S., Feigelman, M. V., Zh. Eksp. Teor. Fiz. 86 (1984) 1544 ; J. Phys. C 16 (1983) L-803.

[2] Chudnovsky, E. M., Serota, R. A., Phys. Rev. B 26 (1982) 2697.

[3] Efetov, K. B., LARKIN, A. I., Zh. Eksp. Teor. Fiz. 72 (1977) 2350.

[4] Villain, J., SÉmÉRIA, B., J. Physique Lett. 44 (1983) L-889.

[5] Villain, J., J. Physique Lett. 43 (1982) L-551.

[6] Grinstein, G., Ma, S.-K., Phys. Rev. B 28 (1983) 2588.

[7] Nattermann, T., in " Proceedings of the XXth Winter School on Theoretical Physics » Karpacz 1984, Lecture Notes in Physics (Springer, Berlin) 1984.

[8] Schwartz, M., Shapir, Y., Aharony, A., Phys. Lett. A 106 (1984) 191.

[9] Villain, J., Séméria, B., Lancon, F., Billard, L., J. Phys. C 16 (1983) 6153.

[10] ImRy, Y., Ma, S.-K., Phys. Rev. Lett. 35 (1975) 1399.

[11] Nattermann, T., J. Phys. C 16 (1983) 6407.

[12] Parisi, G., Sourlas, N., Phys. Rev. Lett. 43 (1979) 744.

[13] Bray, A. J., J. Phys. C 16 (1983) 5879.

[14] Parisi, G., Sourlas, N., Nucl. Phys. B 206 (1982) 321.

[15] Villain, J., Phys. Rev. Lett. 52 (1984) 1543.

[16] Bruinsma, R., Aeppli, G., Phys. Rev. Lett. 52 (1984) 1547.

[17] NatTermanN, T., preprint. 